\title{
Prognostic Aspects of Lupus Nephritis at Aristide Le Dantec University Hospital in Dakar
}

\author{
Mbengue Mansour, Faye Maria, Cissé Mouhamadou Moustapha, Lemrabott Tall Ahmed, \\ Fall Khodia, Keita Alex, Faye Moustapha, Ba Bakary, Diagne Seynabou, Keita Niakhaleen, \\ Ba Mamadou Aw, Dieng Ameth, Motula Latou Lot, Niang Abdou, Diouf Boucar, Ka El Hadji Fary
}

Department of Nephrology, Aristide Le Dantec University Hospital, Dakar, Senegal

Email: mansourmbengue92@gmail.com

How to cite this paper: Mansour, M., Maria, F., Moustapha, C.M., Ahmed, L.T., Khodia, F., Alex, K., Moustapha, F., Bakary, B., Seynabou, D., Niakhaleen, K., Aw, B.M., Ameth, D., Lot, M.L., Abdou, N., Boucar, D. and El Hadji Fary, K. (2018) Prognostic Aspects of Lupus Nephritis at Aristide Le Dantec University Hospital in Dakar. Open Journal of Nephrology, 8, 124-132. https://doi.org/10.4236/ojneph.2018.84014

Received: December 5, 2018

Accepted: December 26, 2018

Published: December 29, 2018

Copyright () 2018 by authors and Scientific Research Publishing Inc. This work is licensed under the Creative Commons Attribution International License (CC BY 4.0).

http://creativecommons.org/licenses/by/4.0/

\section{(c) (i) Open Access}

\begin{abstract}
Introduction: Kidney injury is common in the course of lupus and affects the functional and vital prognosis. The risk of progression to end-stage renal failure can reach $40 \%$ to $60 \%$. Thus we carried out this work for the purpose of an evaluation of the renal and vital prognosis and to deduce the factors of poor prognosis. Patients and method: This was a retrospective, descriptive and analytical study conducted over a period of 10 years from January 1, 2007 to December 31, 2016, performed in the Nephrology Department of Aristide Le Dantec Hospital in Dakar. Patients with lupus nephritis were included. The studied parameters were epidemiological, clinical, paraclinical and progression. We had done a crossover of the patients to look for the factors of poor renal and vital prognosis. Results: Out of 93 cases of lupus patients, 64 were included, a prevalence of $69 \%$. The mean age of the patients was $31.97 \pm$ 10.44 years old. There were $81 \%$ women and $19 \%$ men, a sex ratio of 0.23 . Class III was found in 24 cases (37.5\%), Class IV in 20 cases (31.25\%), Class V in 15 cases $(23.4 \%)$, Class II in 4 cases $(6.25 \%)$ and Class I in 1 case (1.6\%). The combination of corticosteroids and immunosuppressants was used in $56.25 \%$ of cases. After a follow-up of six months, 19 patients were in complete remission, 21 had resistance and 9 had partial remission. Of the 21 patients who had resistance, 8 were in chronic renal failure. Death was observed in 5 patients and the causes were in 3 patients: pulmonary embolism, bacterial meningitis and pulmonary tuberculosis. The cause of death was unknown in 2 patients. The factors of poor renal prognosis were lymphopenia, the presence of anti-native DNA antibodies, nephrotic syndrome, microscopic hematuria, tubular atrophy and interstitial fibrosis. Risk factors affecting renal survival were the presence of native anti-DNA antibodies, microscopic hematuria, leukocyturia and the presence of a proliferative class. The factors of poor prognosis were renal failure, lymphopenia, nephrotic syndrome, glo-
\end{abstract}


merular sclerosis, arteriosclerosis, interstitial infiltration and tubular atrophy. Conclusion: The risk conferred by nephropathy is greater for proliferative glomerulonephritis; it is also correlated with the presence of persistent nephrotic syndrome or severe renal failure.

\section{Keywords}

Remission, Resistance, Chronic Renal Failure, Lupus Nephritis

\section{Introduction}

Kidney injury is one of the most severe manifestations of systemic lupus erythematosus (SLE), affecting 40\% - 60\% of patients with SLE [1] [2]. Two previous studies in Senegal reported a hospital prevalence of 56\% and 72\% [3] [4]. The risk of progression to end stage renal disease (ESRD) is relatively low in all randomized studies published in recent years, estimated at less than $10 \%$ of patients after a follow-up of 5 to 10 years [5]. However, larger epidemiological studies reveal a greater risk when approaching the usual clinical practice, ranging from $19 \%$ in the Caucasian subject to $69 \%$ in the black subject [5]. As for the vital prognosis of lupus patients, it is strongly influenced by the existence or not of lupus nephritis. In a large European cohort [6], it was thus shown that the overall survival, measured at 10 years from the discovery of lupus, was $94 \%$ for patients without nephropathy against $88 \%$ for patients with lupus nephritis [7]. In an earlier study in Senegal, mortality was $9 \%$ in patients with lupus nephritis [3]. Thus, we performed this work for the purpose of evaluating the renal and vital prognosis of lupus nephritis.

\section{Patients and Method}

This was a retrospective, descriptive and analytical study, conducted over a period of 10 years from January 1, 2007 to December 31, 2016 in the nephrology department of Aristide Le Dantec Hospital in Dakar. Patients with lupus nephritis were included. Patients whose laboratory explorations were incomplete were excluded. The diagnosis of lupus nephritis was retained in the presence of a concordant renal biopsy with a proteinuria to creatinuria ratio (PCR) greater than $0.5 \mathrm{~g} / \mathrm{g}$ or proteinuria greather than $0.5 \mathrm{~g} / 24 \mathrm{~h}$ or active urinary sediment [8]. For each selected patient, epidemiological, clinical, biological, histological, therapeutic and evolutionary data were studied. The glomerular filtration rate was estimated according to the modification of Diet in Renal Disease (MDRD) formula. Histological lesions were based on the 2003 ISN/RSP classification.

Complete or partial remission, relapse and resistance were defined according to EULAR/ERA/EDTA criteria [9]:

- The complete remission was defined by a daily proteinuria $<0.5 \mathrm{~g}$ or PCR $<$ $50 \mathrm{mg} / \mathrm{mmol}$ and a normal glomerular filtration rate (GFR) or not falling more than $10 \%$ of normal. 
- Partial remission was defined as a decrease in proteinuria by more than $50 \%$ and a daily proteinuria $<3 \mathrm{~g}$ and by normal or near normal GFR (preferably within 6 months but not after 12 months of induction therapy).

- The resistance was defined by:

- a lack of improvement within 3 to 4 months;

- or an absence of partial remission after 6 to 12 months of treatment;

- or absence of complete remission after 2 years of treatment.

- The relapse was:

- Nephritic if serum creatinine increase greater than or equal to $30 \%$ or GFR decrease greater than or equal to $10 \%$ and active urinary sediment.

- Proteinuric if PCR $>100 \mathrm{mg} / \mathrm{mmol}$ (proteinuria $>1 \mathrm{~g}$ ) after complete remission or $>200 \mathrm{mg} / \mathrm{mmol}$ (proteinuria $>2 \mathrm{~g}$ ) after partial remission.

We crossed over to look for the factors of bad prognosis vital, renal and affecting the renal survival.

By comparing the demographic, clinical, biological, histological and evolutionary data of the patients, we divided them into 2 groups ( $\mathrm{A}$ and $\mathrm{B}$ ) to obtain the factors of bad prognosis vital.

Group A: alive.

Group B: Deceased.

By comparing the demographic, clinical, biological, histological and evolutionary data of the patients, we divided the patients into 2 groups ( $C$ and $D)$ in order to obtain the factors of poor renal prognosis.

Group C: patients who were in complete or partial remission.

Group D: Patients who had made a resistance.

The comparison in univariate analysis of the demographic, clinical, biological and histological parameters of two groups of patients: those who progressed to end-stage chronic kidney desease (CKD) (group E) and those who either normalized their renal function or kept it at CKD stage but not end-stage (group F), in order to retain the risk factors affecting renal survival.

The local ethics committee approved the study.

The data entry was made using the software "The sphinx" version 5.1.0.2.

The analysis of the data was done using SPSS (Statistical Package for Social Science) software version 18 . The averages and percentages were compared using the Student's test and the Chi-square test, and the exact test of Fischer, according to their conditions of applicability.

Any difference below 0.05 was considered statistically significant.

\section{Result}

During the study period, out of 93 cases of lupus patients, 64 patients were included, with a hospital prevalence of $69 \%$. The mean age of the patients was $31.97 \pm 10.44$ years with extremes of 13 and 59 years. The age group between 20 and 29 years was the most represented, found in 29 patients or $45.3 \%$. There were $81 \%$ women and $19 \%$ men, a sex ratio of 0.23 . Class III was found in 24 cases $(37.5 \%)$, class IV in 20 cases $(31.25 \%)$, class V in 15 cases $(23.4 \%)$, class II 
in 4 cases $(6.25 \%)$ and class $I$ in 1 case $(1.6 \%)$. In the induction treatment of patients with proliferative lupus nephritis, cyclophosphamide was used in 19 cases, mycophenolate mofetil in 10 cases and azathioprine in 3 cases. This immunosuppressive treatment was associated with steroids. In patients with class $\mathrm{V}$, cyclophosphamide combined with corticosteroid therapy was used in 4 cases. The other patients received steroids alone. After a follow-up of 6 months, there were 15 lost to follow-up, 19 patients (38.8\%) were in complete remission, 9 patients (18.4\%) were in partial remission, 21 patients $(42.8 \%)$ had resistance. Relapse was observed in $2 \%$ of cases. There are $10.2 \%$ of patients who have had chronic renal failure. Death was observed in 5 patients and the causes were in 3 patients: pulmonary embolism, bacterial meningitis and pulmonary tuberculosis. The cause of death was unknown in 2 patients. The factors of poor renal prognosis were lymphopenia $(p=0.012)$, the presence of native anti-DNA ( $p=0.003)$, the nephrotic syndrome $(\mathrm{p}=0.011)$, microscopic hematuria $(\mathrm{p}=0.015)($ Table 1$)$, tubular atrophy $(\mathrm{p}=0.038)$ and interstitial fibrosis $(\mathrm{p}=0.007)$ (Table 2). Risk factors affecting renal survival were the presence of native anti-DNA antibody ( $p$ $=0.041)$, microscopic hematuria $(\mathrm{p}=0.017)$, leukocyturia $(\mathrm{p}=0.019)($ Table 3$)$ and proliferative class $(\mathrm{p}=0.016)$ (Table 4$)$. The factors of bad prognosis vital were delay between early symptomatology and admission greater than 3 months $(\mathrm{p}=0.045)$, renal insufficiency $(\mathrm{p}=0.001)$, lymphopenia $(\mathrm{p}=0.014)$, nephrotic syndrome $(\mathrm{p}=0.015)$, a delay between early symptomatology and start of treatment greater than 3 months $(\mathrm{p}=0.038)$ (Table 5), glomerular sclerosis $(\mathrm{p}=$ $0.019)$, arteriosclerosis $(p=0.039)$, interstitial infiltration $(p=0.042)$ and tubular atrophy $(\mathrm{p}=0.026)($ Table 6$)$.

\section{Discussion}

The factors of poor renal prognosis were lymphopenia, the presence of anti-native

Table 1. Clinical and biological factors of poor renal prognosis.

\begin{tabular}{cccc}
\hline & Resistance $(\mathbf{n}=\mathbf{2 1})$ & Remission $(\mathbf{n}=\mathbf{2 8})$ & $\mathbf{P}$ \\
Age & $35.4 \pm 7.6$ & $31.2 \pm 8.4$ & 0.564 \\
Sex-ratio & $8 \mathrm{H} / 13 \mathrm{~F}$ & $3 \mathrm{H} / 25 \mathrm{~F}$ & 0.711 \\
Hypertension & 6 cas $(28.57 \%)$ & 6 cas $(21.42 \%)$ & 0.765 \\
Mean serum creatinine & $3.3 \pm 2.7$ & $2.8 \pm 2.2$ & 0.851 \\
Renal insufficiency & 12 cas $(57.14 \%)$ & 7 cas $(25 \%)$ & 0.355 \\
Anemia & 19 cas $(90.47 \%)$ & 24 cas $(85.71 \%)$ & 0.212 \\
Lymphopenia & 3 cas $(14.28 \%)$ & 4 cas $(14.28 \%)$ & 0.012 \\
C3 Hypocomplementemia & 1 cas $(4.76 \%)$ & 0 cas $(0 \%)$ & 0.965 \\
CH50 Hypocomplementemia & 1 cas $(4.76 \%)$ & 0 cas $(0 \%)$ & 0.965 \\
Anti-ENA & 8 cas $(38.09 \%)$ & 12 cas $(42.85 \%)$ & 0.519 \\
Anti-dsDNA & 3 cas $(14.28 \%)$ & 5 cas $(17.85 \%)$ & 0.003 \\
Mean proteinuria & $3.7 \pm 2.9$ & $3.4 \pm 2.6$ & 0.185 \\
Nephrotic syndrome & 11 cas $(52.38 \%)$ & 16 cas $(57.14 \%)$ & 0.011 \\
Microscopic hematuria & 6 cas $(28.57 \%)$ & 12 cas $(42.85 \%)$ & 0.015 \\
Leukocyturia & 4 cas $(19.04 \%)$ & 11 cas $(39.28 \%)$ & 0.786 \\
Delay between early symptomatology and & 19 cas $(90.47 \%)$ & 20 cas $(71.42 \%)$ & 0.655 \\
start of treatment greater than 3 months & & &
\end{tabular}


Table 2. Histological factors of poor renal prognosis.

\begin{tabular}{cccc}
\hline & Resistance $(\mathbf{n}=\mathbf{2 1})$ & Remission $(\mathbf{n}=\mathbf{2 8})$ & $\mathbf{P}$ \\
\hline Proliferative class & 20 cas $(95.23 \%)$ & 15 cas $(53.57 \%)$ & 0.455 \\
Extracapillary proliferation & 12 cas $(57.14 \%)$ & 8 cas $(28.57 \%)$ & 0.874 \\
Percentage of crescent & $28.35 \pm 25.3$ & $27.97 \pm 24.8$ & 0.615 \\
Glomerular sclerosis & 6 cas $(28.57 \%)$ & 6 cas $(21.42 \%)$ & 0.066 \\
Percentage of glomerular sclerosis & $19.3 \pm 11.5$ & $21.8 \pm 14.6$ & 0.134 \\
Intracapillary thrombi & 0 cas $(0 \%)$ & 3 cas $(10.71 \%)$ & 0.566 \\
Fibrinoid necrosis & 0 cas $(0 \%)$ & 1 cas $(3.57 \%)$ & 0.855 \\
Arteriosclerosis & 7 cas $(33.33 \%)$ & 3 cas $(10.71 \%)$ & 0.114 \\
Fibrous endarteritis & 8 cas $(38.09 \%)$ & 7 cas $(25 \%)$ & 0.585 \\
Interstitial infiltration & 7 cas $(33.33 \%)$ & 5 cas $(17.85 \%)$ & 0.478 \\
Tubular necrosis & 1 cas $(4.76 \%)$ & 1 cas $(3.57 \%)$ & 0.35 \\
Tubular atrophy & 13 cas $(61.90 \%)$ & 7 cas $(25 \%)$ & 0.038 \\
Interstitial fibrosis & 6 cas $(28.57 \%)$ & 7 cas $(25 \%)$ & 0.007 \\
\hline
\end{tabular}

Table 3. Clinical and biological factors for progression to CKD end-stage.

\begin{tabular}{cccc}
\hline & $\begin{array}{c}\text { CKD end-stage } \\
(\mathbf{n}=\mathbf{5})\end{array}$ & $\begin{array}{c}\text { Not CKD end-stage } \\
(\mathbf{n}=\mathbf{4 4})\end{array}$ & P \\
\hline Age & $40.5 \pm 5.2$ & $31.6 \pm 8.9$ & 0.555 \\
Sex-ratio & $2 \mathrm{H} / 3 \mathrm{~F}$ & $9 \mathrm{H} / 35 \mathrm{~F}$ & 0.396 \\
Delay between early & 1 cas $(20 \%)$ & 9 cas $(20.45 \%)$ & 0.239 \\
symptomatology and start of & & & \\
treatment greater than 3 months & & & \\
Hypertension & 3 cas $(60 \%)$ & 9 cas $(20.45 \%)$ & 0.091 \\
Mean serum creatinine & $56.7 \pm 12.1$ & $19.2 \pm 9.9$ & 0.785 \\
Renal insufficiency & 5 cas $(100 \%)$ & 14 cas $(31.81 \%)$ & 0.718 \\
Anemia & 5 cas $(100 \%)$ & 38 cas $(86.36 \%)$ & 0.366 \\
Lymphopenia & 1 cas $(20 \%)$ & 6 cas $(13.63 \%)$ & 0.615 \\
C3 Hypocomplementemia & 0 cas $(0 \%)$ & 1 cas $(2.27 \%)$ & 0.448 \\
CH50 Hypocomplementemia & 0 cas $(\%)$ & 1 cas $(2.27 \%)$ & 0.446 \\
Anti-ENA & 1 cas $(20 \%)$ & 19 cas $(43.18 \%)$ & 0.854 \\
Anti-dsDNA & 1 cas $(20 \%)$ & 7 cas $(15.90 \%)$ & 0.041 \\
Mean proteinuria & 3 cas $(60 \%)$ & 24 cas $(54.54 \%)$ & 0.717 \\
Nephrotic syndrome & $3.3 \pm 2.6$ & $3.2 \pm 2.6$ & 0.594 \\
Microscopic hematuria & 3 cas $(60 \%)$ & 15 cas $(34.09 \%)$ & 0.017 \\
Leukocyturia & 2 cas $(40 \%)$ & 13 cas $(29.54 \%)$ & 0.019 \\
\hline
\end{tabular}

Table 4. Histological factors for progression to CKD end-stage.

\begin{tabular}{cccc}
\hline & CKD end-stage $(\mathbf{n}=5)$ & $\begin{array}{c}\text { Not CKD end-stage } \\
(\mathbf{n}=\mathbf{4 4})\end{array}$ & $\mathbf{P}$ \\
\hline Proliferative class & 5 cas $(100 \%)$ & 30 cas $(68.18 \%)$ & 0.016 \\
Extracapillary proliferation & 3 cas $(60 \%)$ & 17 cas $(38.63 \%)$ & 0.692 \\
Percentage of crescent & $25.32 \pm 23.2$ & $27.91 \pm 25.3$ & 0.912 \\
Glomerular sclerosis & 2 cas $(40 \%)$ & 11 cas $(25 \%)$ & 0.175 \\
Percentage of glomerular sclerosis & $19.58 \pm 14.3$ & $21.4 \pm 16.01$ & 0.231 \\
Intracapillary thrombi & 0 cas $(0 \%)$ & 3 cas $(6.81 \%)$ & 0.911 \\
Fibrinoid necrosis & 0 cas $(0 \%)$ & 1 cas $(2.27 \%)$ & 0.833 \\
Arteriosclerosis & 2 cas $(40 \%)$ & 8 cas $(18.18 \%)$ & 0.129 \\
Fibrous endarteritis & 2 cas $(40 \%)$ & 13 cas $(29.54 \%)$ & 0.238 \\
Interstitial infiltration & 0 cas $(0 \%)$ & 12 cas $(27.27 \%)$ & 0.985 \\
Tubular necrosis & 0 cas $(0 \%)$ & 2 cas $(4.54 \%)$ & 0.574 \\
Tubular atrophy & 2 cas $(40 \%)$ & 11 cas $(25 \%)$ & 0.177 \\
Interstitial fibrosis & 2 cas $(40 \%)$ & 11 cas $(25 \%)$ & 0.171 \\
\hline
\end{tabular}


Table 5. Clinical and biological factors of bad prognosis.

\begin{tabular}{cccc}
\hline & $\begin{array}{c}\text { Deceased } \\
(\mathbf{n}=\mathbf{5})\end{array}$ & alive $(\mathbf{n}=44)$ & $\mathbf{P}$ \\
Age & $34.1 \pm 5.33$ & $30.8 \pm 7.21$ & 0.855 \\
Sex-ratio & $2 \mathrm{H} / 3 \mathrm{~F}$ & $9 \mathrm{H} / 35 \mathrm{~F}$ & 0.726 \\
Delay between early symptomatology and & 2 cas $(40 \%)$ & 14 cas $(31.81 \%)$ & 0.038 \\
start of treatment greater than 3 months & & & \\
Delay between early symptomatology and & 1 cas $(20 \%)$ & 14 cas $(31.81 \%)$ & 0.045 \\
admission greater than 3 months & & & 0.091 \\
Hypertension & 4 cas $(80 \%)$ & 13 cas $(29.54 \%)$ & 0.096 \\
Mean serum creatinine & $56.7 \pm 10.5$ & $21.3 \pm 14.8$ & 0.001 \\
Renal insufficiency & 3 cas $(60 \%)$ & 25 cas $(56.81 \%)$ & 0.121 \\
Anemia & 4 cas $(80 \%)$ & 41 cas $(93.18 \%)$ & 0.014 \\
Lymphopenia & 1 cas $(20 \%)$ & 6 cas $(13.63 \%)$ & 0.133 \\
Anti-ENA & 4 cas $(80 \%)$ & 16 cas $(36.36 \%)$ & 0.113 \\
Anti-dsDNA & 2 cas $(40 \%)$ & 6 cas $(13.63 \%)$ & 0.095 \\
Mean proteinuria & $3.2 \pm 2.1$ & $3.7 \pm 3.4$ & 0.015 \\
Nephrotic syndrome & 3 cas $(60 \%)$ & 24 cas $(54.54 \%)$ & 0.139 \\
Microscopic hematuria & 4 cas $(80 \%)$ & 14 cas $(31.81 \%)$ & 0.912 \\
Leukocyturia & 4 cas $(80 \%)$ & 11 cas $(25 \%)$ & \\
\hline
\end{tabular}

Table 6. Histological factors of bad prognosis vital.

\begin{tabular}{cccc}
\hline & Deceased $(\mathbf{n}=\mathbf{5})$ & Alive $(\mathbf{n}=\mathbf{4 4})$ & $\mathbf{P}$ \\
\hline Proliferative class & 5 cas $(100 \%)$ & 44 cas $(100 \%)$ & 0.095 \\
Extracapillary proliferation & 5 cas $(100 \%)$ & 13 cas $(29.54 \%)$ & 0.101 \\
Percentage of crescent & $26.27 \pm 23.6$ & $28.11 \pm 24.59$ & 0.097 \\
Glomerular sclerosis & 5 cas $(100 \%)$ & 10 cas $(22.72 \%)$ & 0.019 \\
Percentage of glomerular sclerosis & $20.6 \pm 15.7$ & $21.9 \pm 16.8$ & 0.109 \\
Intracapillary thrombi & 0 cas $(0 \%)$ & 3 cas $(6.81 \%)$ & 0.925 \\
Fibrinoid necrosis & 0 cas $(0 \%)$ & 1 cas $(2.27 \%)$ & 0.738 \\
Arteriosclerosis & 2 cas $(40 \%)$ & 8 cas $(18.18 \%)$ & 0.039 \\
Fibrous endarteritis & 5 cas $(100 \%)$ & 12 cas $(27.27 \%)$ & 0.088 \\
Interstitial infiltration & 2 cas $(40 \%)$ & 10 cas $(22.72 \%)$ & 0.042 \\
Tubular necrosis & 1 cas $(20 \%)$ & 1 cas $(2.27 \%)$ & 0.645 \\
Tubular atrophy & 2 cas $(40 \%$ & 11 cas $(25 \%)$ & 0.026 \\
Interstitial fibrosis & 0 cas $(0 \%)$ & 13 cas $(29.54 \%)$ & 0.931 \\
\hline
\end{tabular}

DNA antibodies, nephrotic syndrome, microscopic hematuria, tubular atrophy and interstitial fibrosis. These factors were associated with a poor renal prognosis in most series. Nephrotic syndrome was found to be a factor of poor renal prognosis by Hajji, Gassongo-Koumou, Mok and Beji [10] [11] [12] [13]. The nephrotic syndrome is a factor of poor renal prognosis because the high proteinuria which constitutes it is a factor of progression of the renal disease. There is a relationship between the degree of proteinuria and the progression of kidney disease. This fact appears in particular, established for all glomerular nephropathies. Various components of proteinuria are probably involved in the formation of tubulointerstitial lesions and renal sclerosis by their cytotoxic effects on tubular cells, and their pro-inflammatory, profibrotic and hyperplastic effects: albumin, cytokines, growth factors (insulin-growth factor or IGF, transforming growth factor beta or TGF-b), transferrin, complement reactive components. In addition, the reabsorption of proteins by the tubular cells creates tubular lesions 
which are sources of interstitial fibrosis [14].

Microscopic hematuria is a factor of poor renal prognosis because it is associated with the presence of cell proliferation which is a factor of poor renal prognosis [15]. The presence of native anti-DNA antibodies was also found to be a factor in poor renal prognosis in other series [12] [16] [17]. This is explained by the fact that the presence of native anti-DNA antibodies reflects severe glomerular damage [16]. Tubular atrophy and interstitial fibrosis were also found to be factors in poor renal prognosis in other series [12] [16] [17]. Tubular atrophy and interstitial fibrosis are factors of poor renal prognosis because their presence reflects an already advanced renal involvement. However, hypertension and renal failure at diagnosis found as factors of poor renal prognosis in several series [11] [13] [16] were not associated with a poor renal prognosis in our series.

The factors affecting renal survival in our series were the presence of native anti-DNA, microscopic hematuria, leukocyturia, the presence of a proliferative class. Among these factors, the presence of a proliferative class and native anti-DNA has been found to be factors affecting renal survival by Ibrahimi, Hajji and Mahmoud [10] [17] [18]. In fact, kidney survival depends on histological lesions. It is in certain series better in classes II and III than in class IV and in other series, equivalent between classes III and IV [13]. Proliferative classes are indisputably those with the poorest prognosis in the medium-dated [15], especially for forms associated with extensive extracapillary proliferation. Hematuria is associated with poor renal survival because it is linked to the existence of cell proliferation.

The factors of bad prognosis were: delay between early symptomatology and admission greater than 3 months, renal insufficiency, lymphopenia, nephrotic syndrome, a delay between early symptomatology and early treatment above 3 months, glomerular sclerosis, arteriosclerosis, interstitial infiltration and tubular atrophy. Renal failure was one of the factors of poor prognosis vital found in our series and has been found in the literature as a factor associated with death in the Hajji and Ibrahimi series [10] [18]. This mortality is linked to the complications of renal failure. Chronic lesions such as glomerular sclerosis, arteriosclerosis and tubular atrophy are poor prognosis vital factors because their presence reflects the existence of a CKD which by these metabolic complications and its susceptibility to infections, increases the risk of death. A delay between early symptomatology and admission over 3 months and a delay between early symptomatology and start of treatment greater than 3 months are poor prognosis vital factors because they are related to the lateness of care and therefore facilitates the appearance of CKD and its complications. The nephrotic syndrome by its thromboembolic and infectious complications increases the risk of death (in our series the death was observed in 5 patients and the causes were in 3 patients: a pulmonary embolism, a bacterial meningitis and a pulmonary tuberculosis). 


\section{Conclusion}

The risk conferred by nephropathy is greater for proliferative glomerulonephritis. It is also correlated with the presence of persistent nephrotic syndrome or severe renal insufficiency.

\section{Conflicts of Interest}

The authors declare that there is no conflict of interest regarding the publication of this paper.

\section{References}

[1] Feldman, C.H., Hiraki, L.T., Liu, J., et al. (2013) Epidemiology and Socio-Demographics of Systemic Lupus Erythematosus and Lupus Nephritis among US Adults with Medicaid Coverage, 2000-2004. Arthritis \& Rheumatism, 65, 753-763. https://doi.org/10.1002/art.37795

[2] Seligman, V.A., Lum, R.F., Olson, J.L., et al. (2002) Demographic Differences in the Development of Lupus Nephritis: A Retrospective Analysis. The American Journal of Medicine, 112, 726-729. https://doi.org/10.1016/S0002-9343(02)01118-X

[3] Ka, E.F., Cisse, M.M., Lemrabott, A.T., Fall, S., Diallo, M., Diallo, S., Faye, M., Niang, A. and Diouf, B. (2013) Néphropathie lupique chez les sujets génétiquement pigmentés vivant au Sénégal: A propos de quarante-trois cas. Médecine et Santé Tropicales, 23, 328-331.

[4] Niang, A., Ka, E.F., Dia, D., Pouye, A., et al. (2008) Lupus Nephritis in Senegal: A Study of 42 Cases. Saudi Journal of Kidney Diseases and Transplantation, 19, 470-474.

[5] Adler, M., Chambers, S., Edwards, C., et al. (2006) An Assessment of Renal Failure in an SLE Cohort with Special Reference to Ethnicity, over a 25-Year Period. Rheumatology(Oxford), 45, 1144-1147. https://doi.org/10.1093/rheumatology/kel039

[6] Cervera, R., Khamashta, M.A., Font, J., et al. (2003) Morbidity and Mortality in Systemic Lupus Erythematosus during a 10-Year-Period: A Comparison of Early and Late Manifestations in a Cohort of 1000 Patients. Medicine (Baltimore), 82, 299-308. https://doi.org/10.1097/01.md.0000091181.93122.55

[7] Mok, C.C., Kwok, R.C. and Yip, P.S. (2013) Effect of Renal Disease on the Standardized Mortality Ratio and Life Expectancy of Patients with Systemic Lupus Erythematosus. Arthritis \& Rheumatism, 65, 2154-2160.

https://doi.org/10.1002/art.38006

[8] Hahn, B.H., McMahon, M.A., Wilkinson, A., et al. (2012) American College of Rheumatology Guidelines for Screening, Treatment, and Management of Lupus Nephritis. Arthritis Care \& Research, 64, 797-808. https://doi.org/10.1002/acr.21664

[9] Bertsias, G.K., Tektonidou, M., Amoura, Z., et al. (2012) Joint European League Against Rheumatism and European Renal Association-European Dialysis and Transplant Association (EULAR/ERA-EDTA) Recommendations for the Management of Adult and Paediatric Lupus Nephritis. Annals of the Rheumatic Diseases, 71, 1771-1782. https://doi.org/10.1136/annrheumdis-2012-201940

[10] Hajji, M., Harzallah, A., Barreh, S., et al. (2015) Facteurs pronostiques des néphropathies lupiques prolifératives. Néphrologie \& Thérapeutique, 11, 338-406. https://doi.org/10.1016/j.nephro.2015.07.360 
[11] Gassongo-Koumou, G.C., Soumeila, I. and Eyeni Sinomono, D.T. (2015) Néphropathie lupique: Impact de l'atteinte tubulo-interstitielle dans le pronostic fonctionnel rénal. Néphrologie \& Thérapeutique, 11, 338-406. https://doi.org/10.1016/j.nephro.2015.07.272

[12] Mok, C.C. (2005) Prognostic Factors in Lupus Nephritis. Lupus, 14, 39-44. https://doi.org/10.1191/0961203305lu2057oa

[13] Béji, S., Kaaroud, H., Ben Moussa, F., et al. (2005) Néphropathie lupique: A propos de 211 cas. La Revue de Médecine Interne, 26, 8-12. https://doi.org/10.1016/j.revmed.2004.09.003

[14] Peraldi, M.N. (2014) Néphropathies glomérulaires. In: Kanfer, A., Kourilsky, O., Peraldi, M.N. and Combe, C., Eds., Néphrologie et Troubles Hydro-électrolytiques, Elsevier Masson, 1-59. https://doi.org/10.1016/B978-2-294-73759-6.00001-8

[15] Korbet, S.M., Lewis, E.J., Schwartz, M.M., et al. (2000) Factors Predictive of Outcome in Severe Lupus Nephritis. American Journal of Kidney Diseases, 35, 904-914. https://doi.org/10.1016/S0272-6386(00)70262-9

[16] Safari, J. (2015) Néphropathie lupique: Etude descriptive à propos de 553 cas et éléments pronostiques à 12 mois de suivi au CHR des Grands-Lacs. Néphrologie \& Thérapeutique, 11, 338-406. https://doi.org/10.1016/j.nephro.2015.07.267

[17] Mahmoud, G.A., Zayed, H.S. and Ghoniem, S.A. (2015) Renal Outcomes among Egyptian Lupus Nephritis Patients: A Retrospective Analysis of 135 Cases from a Single Centre. Lupus, 24, 331-338. https://doi.org/10.1177/0961203314567751

[18] Ibrahimi, A., Elhoussni, S., Rhou, H., et al. (2015) Néphropathie lupique: Caractéristiques et facteurs pronostiques (à propos d'une cohorte de 252 patients). Néphrologie \& Thérapeutique, 11, 338-406. https://doi.org/10.1016/j.nephro.2015.07.354 